\title{
Tuning thermal transport in graphene via combinations of molecular antiresonances
}

\author{
Koray Sevim a , Hâldun Sevinçli b, * \\ a Department of Physics, Izmir Institute of Technology, 35430, Urla, Izmir, Turkey \\ b Department of Materials Science and Engineering, Izmir Institute of Technology, 35430, Urla, Izmir, Turkey
}

\section{A R T I C L E I N F O}

Article history:

Received 16 April 2018

Received in revised form

17 August 2018

Accepted 25 August 2018

Available online 1 September 2018

\begin{abstract}
A B S T R A C T
We propose a method to engineer the phonon thermal transport properties of low dimensional systems. The method relies on introducing a predetermined combination of molecular adsorbates, which give rise to antiresonances at frequencies specific to the molecular species. Despite their dissimilar transmission spectra, thermal resistances due to individual molecules remain almost the same for all species. On the other hand, thermal resistance due to combinations of different species are not additive and show large differences depending on the species. Using a toy model, the physics underlying the violation of resistance summation rule is investigated. It is demonstrated that equivalent resistance of two scatterers having the same resistances can be close to the sum of the constituents or $\sim 70 \%$ of it depending on the relative positions of the antiresonances. The relative positions of the antiresonances determine the net change in transmission, therefore the equivalent resistance. Since the entire spectrum is involved in phonon spectrum changes in different parts of the spectrum become important. Performing extensive first-principles based computations, we show that these distinctive attributes of phonon transport can be useful to tailor the thermal transport through low dimensional materials, especially for thermoelectric and thermal management applications.
\end{abstract}

() 2018 Elsevier Ltd. All rights reserved.

\section{Introduction}

Graphene has not only peculiar electronic properties but unique phononic and thermal properties as well [1,2]. It is required to have control over its phonon transport properties for efficient thermal and thermoelectric applications. There are several proposals for that purpose, some of which are reducing the dimension by fabricating nano-ribbons [3], introducing defects [4] and edge-shape disorder [5,6], including isotopes with different distributions such as random atomic distributions and cluster formations [7], geometrical structuring, imposing out-of-plane deformations [8], hybrid schemes of geometrical structuring and isotope clusters [9], and clamping the out-of-plane modes by molecular functionalization [10]. Apart from these, interference, a fundamental aspect of wave-propagation, has immense effects on transport, especially at the nano-scale. Antiresonance and Fano lineshapes are manifestations of interference and they have been major topics in electronic transport in recent years [11-16]. In electronic transport, only the

\footnotetext{
* Corresponding author.

E-mail address: haldunsevincli@iyte.edu.tr (H. Sevinçli).
}

features close to the Fermi level affect the conductance, independent of the richness of interference patterns in the rest of the spectrum. On the other hand, phonon transport involves the whole spectrum, most of the contribution to thermal current coming form the low-energy acoustic modes. Recently, phononic crystals which rely on phonon interferences were fabricated $[18,19]$, two-photon interference principle was shown to be applicable to phonon transport [20]. It was also shown that structural resonances [21], ring-type structures [22] and three-dimensional architectures [23] can be engineered to control phonon transmission via interference effects. We should distinguish between two types of interferences. One is due to multiple reflections between scatterers. At the dilute scatterer limit, this is expected to have minor effect on total transmission. The second one takes place due to coupling to the adsorbate's vibrational modes, which is at the primary focus of the present study.

Here, it is shown that antiresonances introduced in the transmission spectrum upon molecular functionalization can be tuned by suitable choice of molecular species, and that it is possible to engineer thermal transport by choosing suitable combinations of scatterers. We investigate the conductance values for a large set of 
possible combinations, and demonstrate that it is possible to engineer thermal transport by using ensembles of molecular adsorbates. In order to do that, we first investigate a toy model. Then, we simulate realistic systems using the density functional theory based tight binding (DFTB) and atomistic Green function (AGF) methods (see Fig. 1a-b). Having obtained the transmission spectra (TS) for individual scatterers, we employ scaling theory within the cascade scattering approximation to compute the TS for different ensembles of scatterers. We show that, resistance summation rule does not apply to phonon transport and that this opens a way to engineer thermal transport.

\section{Toy model}

We use a toy model to explore the fundamental features of phononic antiresonance lineshapes upon molecular functionalization. Interference due to side groups has been widely studied in electron transport [11-14]. Inspired by those studies, we consider a monatomic linear chain with a side group (see Fig. 1b). The left and the right parts are considered as semi-infinite pristine hot and cold reservoirs, whereas the central part is the scattering region. For the sake of simplicity, all masses are assumed to be equal $(m)$ and all spring constants are equal to $k_{1}\left(k_{1}=m \omega_{1}^{2}\right)$ except the one that binds the side group to the chain $\left(k_{2}=m \omega_{2}^{2}\right)$. The retarded Green function of the scattering region can be expressed as $G(\omega)=$ $\left(\omega^{2}-2 \omega_{1}^{2}-\omega_{2}^{2}-\Sigma_{L}-\Sigma_{R}-\Sigma_{S}\right)^{-1}$, where $\Sigma_{L / R}$ are the self energy functions due to coupling to the reservoirs and $\Sigma_{S}$ is that due to the side group [24]. $\Sigma_{L / R}$ are continuous functions up to $\omega_{\max }$, whereas $\Sigma_{S}$ has discontinuities because of its discrete vibrational spectrum. The lowest resonance in the DOS is due to the zero frequency translational mode of the free molecule, which is shifted due to coupling to the reservoir. Higher frequency resonances are due to internal degrees of freedom of the molecule. These vibrations are not well hybridized with the chain's modes for $\omega_{2}=0.2 \omega_{1}$, and they stay localized on the molecule as a result of interference. Antiresonance line shapes (see Fig. 1c) are due to these localized modes and their frequencies and widths are determined mainly by two factors; namely the internal degrees of freedom of the side group and the bond strength. We first study the simplest case by including a single atom as the side group, in which the transmission spectrum can be obtained analytically as $\zeta(\omega)=1-\frac{\gamma^{2}}{\left(\omega^{2}-\omega_{2}^{2}\right)^{2}+\gamma^{2}}$,

with $\gamma=\omega \omega_{2}^{2} / \sqrt{4 \omega_{1}^{2}-\omega^{2}}$. (see Supplemental Material for details) $\zeta(\omega)$ has an antiresonance at $\omega_{2}$ with an inverted Lorentzian-like shape. At the weak coupling limit, i.e. $\omega_{2} / \omega_{1} \ll 1$, one can approximate $\gamma \approx \omega_{2}^{3} / 2 \omega_{1}$ around the antiresonance and the full width of the antiresonance at half of the pristine value can be approximated as to $w=\omega_{2}\left(\eta+\eta^{3} / 8\right)$, with $\eta=\omega_{2} / 2 \omega_{1}$. Single-atom side group model captures the main features of the fundamental vibration of an adsorbed molecule on a surface. When the side group is weakly coupled to the system, the width is proportional to $k_{2}$ and inversely proportional to $k_{1}^{1 / 2}$. Stronger coupling opens a wider antiresonance in the TS. But the position of the antiresonance shifts to higher frequencies, whose contributions to conductance are less.

The vibrational modes of multi-atom side groups give rise to more detailed interference patterns. The width of the antiresonance depends not only on the strength of adsorption. The vibrational frequency of the mode, as well as the contribution of the linking atoms on the molecular mode play a role. It should also be noted that the line shapes are not always symmetric but Fano-type asymmetric dips are also predicted from the toy model (see Fig. 1c). The angles between the adsorption bond and the displacement direction of the end atom for that particular mode are additional factors that determine the antiresonance lineshapes, which are not addressed in the toy model but are treated exactly in the simulations. Moreover, interference between internal degrees of freedom induced by coupling to the surface might play a role, as well [25]. All these effects are included in an exact way in our simulations.

\section{First principles based simulations}

We simulate graphene, which is functionalized with carbon chains $\mathrm{C}_{n} \mathrm{H}_{m}$ of different lengths $(1 \leq n \leq 5)$ and with aromatic molecules consisting of different numbers of benzene rings, namely benzene, biphenyl and (para-)terphenyl (see Fig. 1). The carbon chains are passivated with hydrogen atoms at their ends, and $m$ equals to 3 or 1 depending on whether $n$ is odd or even, respectively. The simulations are carried out using the DFTB method [26-29]. The periodic boundary conditions are applied in both $x$ and $y$-directions. The simulation cell is taken large enough in both directions to ensure that inter-molecular interactions are (a)

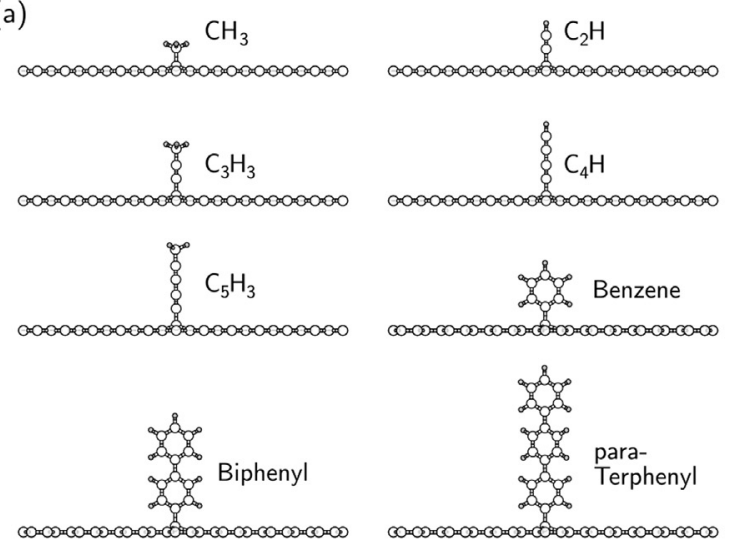

(b)

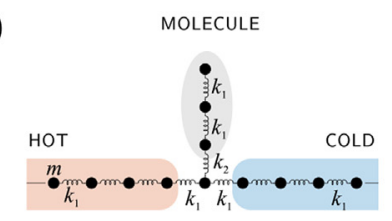

(c)

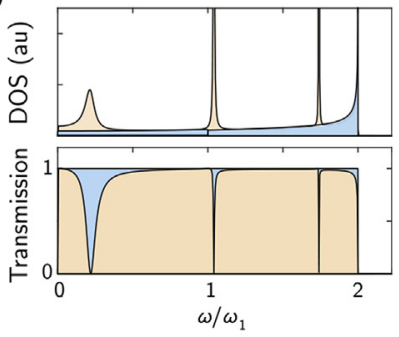

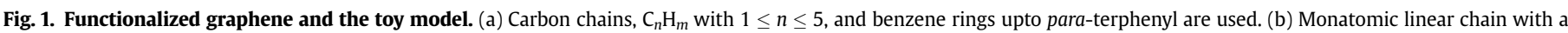

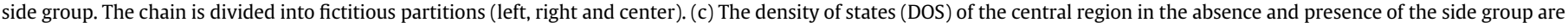

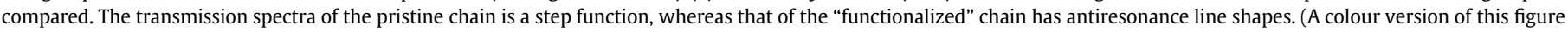
can be viewed online.) 
negligible. Namely, the rectangular super cell contains 432 carbon atoms for the graphene layer and has a size of $38.5 \AA$ by $29.7 \AA$ with an interlayer spacing of $32 \AA$. Carbon atoms close to the boundaries of the simulation cell are fixed at their initial positions so that the force constants between the scattering region and the reservoirs are identical to those of the pristine graphene, a necessary condition for correctly linking the reservoirs to the scattering region. In all structures, the side groups are covalently bonded from their end atoms to a carbon atom of graphene, whose bond lengths and bond angles resemble that of $\mathrm{sp}^{3}$ hybridized carbon. The force constant matrix of the scattering region and those of the reservoirs are computed separately using finite displacement method [30]. TS and DOS are computed using the atomistic Green function (AGF) method as explained in the Supplemental Materials.

DOS and transmission spectra of functionalized structures follow the same trends with pristine graphene in the entire spectrum, with additional peaks in the DOS and corresponding dips in the transmission, as shown in Fig. 2a. The association of a transmission dip with a resonance in the DOS is evident in the inset of Fig. 2a, where two transmission dips at $30 \mathrm{~cm}^{-1}$ and $200 \mathrm{~cm}^{-1}$ for $\mathrm{C}_{3} \mathrm{H}_{3}$ and one at $65 \mathrm{~cm}^{-1}$ for $\mathrm{C}_{2} \mathrm{H}$ are shown. As a matter of fact, similar dips and peaks in DOS and transmission were already observed in the toy model due to interference between the modes of the side group and the chain. A comparison of the localized modes with the extended ones is possible in the Supplemental Videos, where two vibrational modes, one localized and one extended, are visualized for $\mathrm{C}_{3} \mathrm{H}_{3}$. The modes have approximately the same energies around $200 \mathrm{~cm}^{-1}$.

The details of the transmission and DOS of the studied structures can be found in Fig. S1. The number of sharp resonances in the DOS increases with the number of atoms of the side group. Some of the localized modes can be directly matched with distinct transmission antiresonances, eg. the modes around $65 \mathrm{~cm}^{-1}$ and 540 $\mathrm{cm}^{-1}$ of $\mathrm{C}_{2} \mathrm{H}$ functionalized graphene, whereas the effect on the TS is not as clearly distinct for some of the localized modes. It is evident that the peaks below $400 \mathrm{~cm}^{-1}$ in the DOS of biphenyl and para-terphenyl functionalized graphene are responsible for the reduction of transmission in the same frequency range but the lineshapes are not as sharp. It is worth emphasizing that molecular species determines the TS and different species give rise to completely different sets of antiresonances.

Next, the effects of functional groups on conductance are investigated as a function of temperature. Thermal conductance is calculated using the Landauer approach with [31].
$\kappa(T)=\frac{k_{B}}{2 \pi} \int d \omega p(\omega, T) \zeta(\omega)$,

where $T$ is temperature, $k_{B}$ is Boltzmann constant [31]. Transmission spectrum $(\zeta)$ and thermal conductance $(\kappa)$ are normalized with the width of the simulation cell. The weight function is defined by using Bose-Einstein distribution function as $p(x)=-$ $x^{2} \partial f_{B E} / \partial x$, where $f_{B E}=\left(e^{x}-1\right)^{-1}$ and $x=\hbar \omega / k_{B} T$. A note on the weight function is in order here. At low frequencies, $\lim _{\omega \rightarrow 0} p=1$, independent of temperature and it decreases monotonically with increasing frequency. This is one of the reasons for the domination of acoustic modes in thermal transport. At high temperatures, the weights of all modes equalize, that is $p \simeq 1$ for $k_{\mathrm{B}} T / \hbar \omega_{\max } \gg 1$.

In Fig. $2 \mathrm{a}-\mathrm{b}, \zeta$ and $\kappa$ values are plotted for different functional groups and compared against that of pristine graphene. Even though the transmission spectra include a richness of features depending on the molecular species, $\kappa$ values are almost the same in the entire temperature range. At room temperature, the difference between the maximum and the minimum of $\kappa$ values is only $0.03 \mathrm{nW} \mathrm{K}^{-1} \mathrm{~nm}^{-1}$, which is about $2.5 \%$ of the average value, whereas the variance is $\sigma^{2}=10^{-4} \mathrm{nW}^{2} \mathrm{~K}^{-2} \mathrm{~nm}^{-2}$. Namely, the fine structure in the transmission spectra are averaged out within the conductance integral. In electron transport, antiresonances affect electrical conductance significantly, which is the key for many sensor applications, but the bosonic nature of phonons make it impossible to detect the fine details of the spectra in the conductance.

Besides the fact that thermal resistances of the studied side groups are almost equal, their combinations generate large variances, which is also an outcome of the bosonic statistics. In wave mechanics, it is well known that resistances due to two distinct scatterers can not be added in the coherent regime (see e.g. Ref. [32]) but it is possible to combine scatterers to obtain the overall transmission in the quasi-ballistic and diffusive regimes according to the scaling theory. [32-34] One can show that the reduction in conductance due to two antiresonances is larger when the antiresonances are distinct rather than having equal frequencies.

Transmission due to $n$ scatterers can be calculated using the cascade scattering approximation $[35,36]$ as

$\frac{1}{\zeta}=\frac{1}{\zeta_{o}}+\sum_{i} n_{i}\left(\frac{1}{\zeta_{i}}-\frac{1}{\zeta_{o}}\right)$,
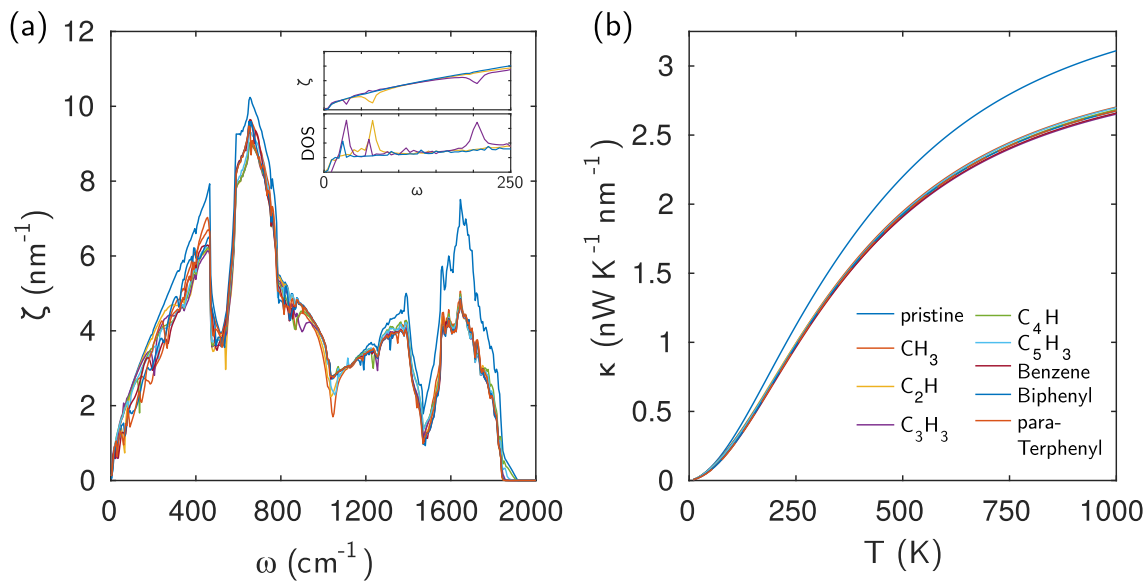

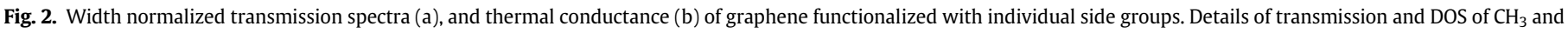
$\mathrm{C}_{2} \mathrm{H}$ are included in the inset of the left panel. (A colour version of this figure can be viewed online.) 
where $\zeta_{o / i}$ is the transmission of the pristine system or due to the side group $i$ and $n_{i}$ is the number of those side groups, $n=\sum_{i} n_{i}$. For electrons at zero temperature, Equation (3) reproduces the summation rule for series of resistances, $\Omega_{\mathrm{eq}}=\sum_{i} \Omega_{i}$. For phonons, thermal resistance $(R=1 / \kappa)$ violates the summation rule (see Equation (2)).

Violation of the resistance summation rule can be demonstrated by investigating the case of two scatterers. For the sake of simplicity we assume that individual scatterers' transmission spectra are different from the pristine case $\left(\zeta_{0}\right)$ only around the antiresonant frequencies. We consider two cases, (i) when the antiresonant frequencies are distinct $\omega_{1} \neq \omega_{2}$, with corresponding transmission spectra $\zeta_{1}$ and $\zeta_{2}$ (see Fig. 3a-b); (ii) when they are equal $\omega_{1}=$ $\omega_{2}=\omega^{\prime}$, and the corresponding transmission spectrum for a single scatterer is $\zeta^{\prime}$ (see Fig. 3d). The total transmission, $\zeta$, due to two scatterers can be written in the cascade scattering approximation as (cf. Equation (3))

$\frac{1}{\zeta / \zeta_{o}}=1+\frac{1}{z_{1}}+\frac{1}{z_{2}}$

where $z_{i}^{-1}=\zeta_{0} / \zeta_{i}-1$. Here, the function $1 / z_{i}$ is sharply peaked around $\omega_{i}$, and approximately equal to zero otherwise. It is important to note that, when the antiresonances are distinct, i.e. $\omega_{1} \nsim \omega_{2}$ and narrow $\left(\Delta \omega \ll \omega_{1}, \omega_{2}\right)$ one can approximate the transmission as $\zeta / \zeta_{o}=1-\left(1+z_{1}\right)^{-1}-\left(1+z_{2}\right)^{-1}$ (see Fig. 3c) This expression enables us to analyze the resistance summation rule as follows. Assuming that the widths of the antiresonances are equal and that the temperature is high enough such that the prefactor $p$ is almost constant around $\omega_{i}$ within $\Delta \omega$, one can write

$\kappa_{\left(\omega_{1} \neq \omega_{2}\right)} \simeq \frac{k_{B}}{2 \pi} \int d \omega \zeta_{\mathrm{o}}\left(p-\frac{p_{1}}{1+z_{1}}-\frac{p_{2}}{1+z_{2}}\right)$.

One notes that $\kappa_{0}=k_{\mathrm{B}} / 2 \pi \int d \omega p \zeta_{0}$ is the pristine conductance. Here, reductions due to distinct antiresonances are additive. When the antiresonances are at the same frequency, $\omega_{1}=\omega_{2}=\omega^{\prime}$, that is when $z_{1}=z_{2}=z^{\prime}$, one has $\zeta / \zeta_{o}=1-2\left(2+z^{\prime}\right)^{-1}$ and

$\kappa_{\left(\omega_{1}=\omega_{2}=\omega^{\prime}\right)} \simeq \frac{k_{B}}{2 \pi} \int d \omega \zeta_{\mathrm{o}}\left(p-\frac{2 p^{\prime}}{2+Z^{\prime}}\right)$.

The factors $p_{i} /\left(1+z_{i}\right)$ and $2 p^{\prime} /\left(2+z^{\prime}\right)$ are then the effective weight factors standing for reduction in conduction due to resistors. In Fig. 3e, transmission reductions are seen when there are one and two antiresonances at $\omega^{\prime}$. $p_{i}$ can be taken out of the integral as their contributions are only around the antiresonance frequencies, where they are almost constant (see Fig. 3(a and b)). At high temperatures, all modes have equal weights, $p_{1} \simeq p_{2} \simeq 1$, and the ratio of the effective weight factors is larger than 1 for all $\omega$, namely $\left(2+z^{\prime}\right) /\left(1+z_{1}\right)>1$. This inequality means that the reduction is larger when the antiresonances are distinct.

Now, we investigate the effect quantitatively. We choose three scatterers $\left(s_{1}, s_{2}\right.$ and $\left.s_{3}\right)$ with corresponding Lorentzian antiresonances centered at $\omega_{1}=\omega_{\max } / 4, \omega_{2}=\omega_{\max } / 2, \omega_{3}=3 \omega_{\max } / 4$ with half widths at half minima $\Delta \omega=0.01 \omega_{\max }$. At the high temperature limit $(p=1)$ the reduction in conductance due to a single scatterer is $2.96 \%$, independent of the antiresonant frequency. Namely their resistances are equal, $R\left(s_{1}\right)=R\left(s_{2}\right)=R\left(s_{3}\right)=$ $0.032 R_{0}$, where $R_{0}=k_{B} \omega_{\max } / 2 \pi$ is the contact resistance. The critical cases are when two scatterers are present. When the antiresonances are distinct, e.g. $s_{1}$ and $s_{3}$ (Fig. $3 \mathrm{a}-\mathrm{c}$ ), the equivalent resistance is $R\left(s_{1}+s_{3}\right)=0.066 R_{0} \simeq R\left(s_{1}\right)+R\left(s_{3}\right)$, and the conductance reduction is $6.05 \%$. When the antiresonant frequencies are equal, e.g. both scatterers are $s_{2}$ type (Fig. $3 \mathrm{~d}-\mathrm{e}$ ), the equivalent resistance is $R\left(s_{2}+s_{2}\right)=0.046 R_{0}<2 R\left(s_{2}\right)$, and conductance reduction is only $4.24 \%$. This is one of the major findings of the present work, which proves that phonon conduction violates resistance summation rule.

We should emphasize that the violation is more apparent when the reduction in transmission occurs is due to sharp antiresonances rather than an overall reduction. At lower temperatures, when $p_{i}$ (a)

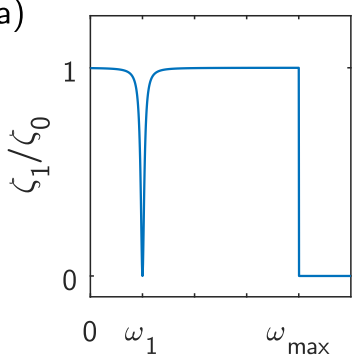

(d)

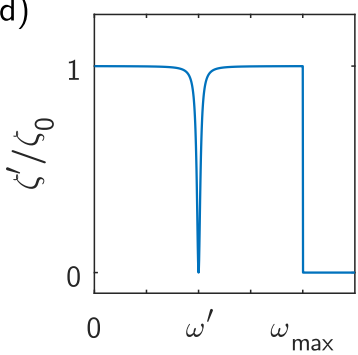

(b)

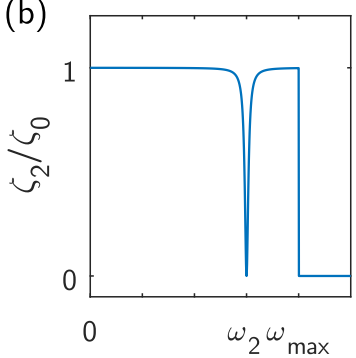

(e)

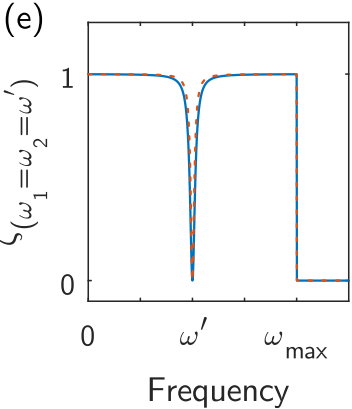

(c)

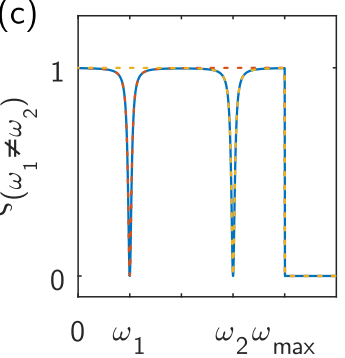

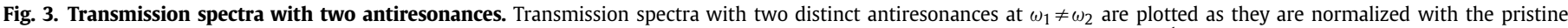

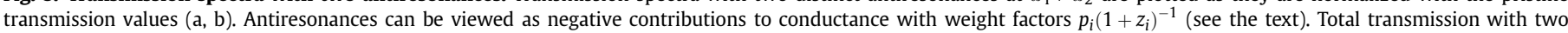

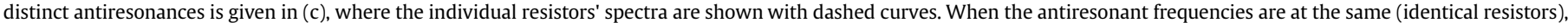

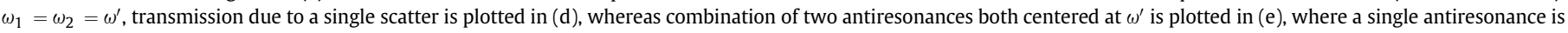
also shown for comparison (dashed). (A colour version of this figure can be viewed online.) 
differ considerably, the ratio of prefactors is $\left(p_{1}+p_{2}\right)\left(2+z^{\prime}\right) / 2 p^{\prime}\left(1+z_{1}\right)$ and the same inequality is achieved if $p_{1}+p_{2} \geq 2 p^{\prime}$. Otherwise, relative values of $z_{i}$ are determinant.

We should mention that the resistance summation is an exact rule for electrons only at zero temperature, that is when transport is truly monochromatic. At finite temperatures, it is a better approximation for electron than for phonons, simply because electron transport takes place around a small energy window around the Fermi energy.

Violation of the summation rule, in fact, paves a way for tuning thermal transport. The overall transmission spectrum can be tailored by using different combinations of molecular adsorbants. In order to investigate the extend of tunability, we compute $\kappa$ of graphene samples, each having 100 chemisorbed molecules. Total transmission is calculated using transmission spectra of individual molecules within the cascade scattering approximation in Equation (3). The molecular species are those shown in Fig. 1. For the sake of simplicity, the molecules are grouped and distributed in bundles, each bundle containing 10 molecules of one species, namely $n_{i}$ are either zero or multiples of 10 . Ensembles are formed by restricting the number of species $\left(N_{\mathrm{sp}}\right)$ to different values ranging from 1 to 8 . Therefore the numbers of possible combinations are 8, 252, 2016, $5880,7056,3528,672$, and 36 , respectively. Afterwards, TS and $\kappa$ are computed for each combination. In Fig. $4, \kappa$ values are plotted for different $N_{\mathrm{sp}}(\mathrm{a}-\mathrm{h})$. The gray curves correspond to individual configurations, the colored curves are their mean values, and the standard deviations are indicated with vertical bars. In Fig. 4i, average $\kappa$ values for different $N_{\mathrm{sp}}$ are compared. In Table 1, minimum and maximum $\kappa$ values, the standard deviation $(\sigma)$ and the ratio of $\sigma$ to the average $\left(\sigma / \kappa_{\mathrm{av}}\right)$ are tabulated for different $N_{\mathrm{sp}}$ at $T=$ 100,300 , and $750 \mathrm{~K}$.

For $N_{\mathrm{sp}}=1$, the combined resistances are expected to be similar, because the resistances of single molecules are almost the same (see Figs. 2(b) and 4(a)). However, this is not fulfilled at low temperatures. At $100 \mathrm{~K}$, the ratio of standard deviation to average conductance $\sigma / \kappa_{\mathrm{av}}$ is 0.527 . This is because smaller number of modes contribute to transport at low temperatures. At higher temperatures, as the number of contributing modes is larger, fine details become less important. At room temperature, $\sigma / \kappa_{\mathrm{av}}$ becomes as low as 0.066 . That is, at room temperature and higher temperatures, one can alter $\kappa$ by $5-10 \%$ for the mono-species case (see Table 1 ). At $300 \mathrm{~K}, \kappa_{\min }$ and $\kappa_{\max }$ can be reduced by $31 \%$ and
$40 \%$, respectively, by changing $N_{\mathrm{sp}}$ from 1 to 8 . As a result, $\kappa$ values ranging between $0.144 \mathrm{nW} \mathrm{K}^{-1} \mathrm{~nm}^{-1}\left(N_{\mathrm{sp}}=4,5,6,7\right)$ and 0.257 $\mathrm{nW} \mathrm{K} \mathrm{K}^{-1} \mathrm{~nm}^{-1}\left(N_{\mathrm{sp}}=1\right)$ are possible, which means a range to mean ratio of 0.66 . At $500 \mathrm{~K} \kappa$ lies between 0.257 and $0.450 \mathrm{nW} \mathrm{K}^{-1} \mathrm{~nm}^{-1}$, with a range to mean ratio of 0.55 ; at $750 \mathrm{~K} \kappa$ lies between 0.331 and $0.581 \mathrm{nW} \mathrm{K}^{-1} \mathrm{~nm}^{-1}$, with the same range to mean ratio of 0.55 . At $100 \mathrm{~K}$, on the other hand, the range is larger than the average value by a factor of 1.46 . The number of molecules in the bundles have a minor effect on the $\kappa$ ranges. When $N_{\mathrm{sp}}$ is close to the number of bundles, the number of possible configurations is reduced. Hence, there is less room for controlling $\kappa$ values. This is reflected in the reduced $\sigma$ values and narrowed ranges for larger $N_{\mathrm{sp}}$ configurations. Quantitatively, for $N_{\mathrm{sp}}=8, \kappa_{\max }$ is increased by $7 \%$, while the $\kappa_{\min }$ is reduced by $1 \%$, when the bundle size is reduced to 5 from 10 . These findings display the tunability of thermal transport upon changing the combinations of species, whose individual thermal resistances are almost identical for all temperatures.

The high range to mean value ratios indicate the possibility of obtaining desired $\kappa$ values by using appropriate combinations of functional molecular species. (i) As it was shown with the analytical calculations above, having distinct antiresonances results in better suppression of transport. This is verified with the simulation results, namely lowest $\kappa$ values are achieved for $N_{\mathrm{sp}}>4$, and highest $\kappa$ are due to $N_{\mathrm{sp}}=1$. (ii) Another important factor is obtaining antiresonances at low frequencies. Since $p(\omega, T)$ (see Equation (2)) decreases monotonically with $\omega$, suppression of acoustic modes is crucial for lowering the $\kappa$. One of the lowest lying antiresonances is expected to originate from the rigid vibration of the molecule with respect to the surface, and its frequency depends on the interaction strength and the molecular mass. Heavier molecules with weaker molecule-surface coupling reduces the frequency of this antiresonance. (iii) Another effect of the interaction strength is on the width of the antiresonance. Since $\gamma \sim k_{2}^{3 / 2}$ (cf. Equation (1)), stronger coupling results in wider antiresonance shapes. (iv) The number of resonances is also a factor in reducing $\kappa$, which is related to the number of internal degrees of freedom of the adsorbant. (v) For nano-scale materials, where the anharmonic mean-free-path is longer than the sample size, phonon-phonon interactions are not expected to play a role. For larger samples, the antiresonant frequencies should be low for the effect to be pronounced, because of longer mean-free-paths at lower frequencies.

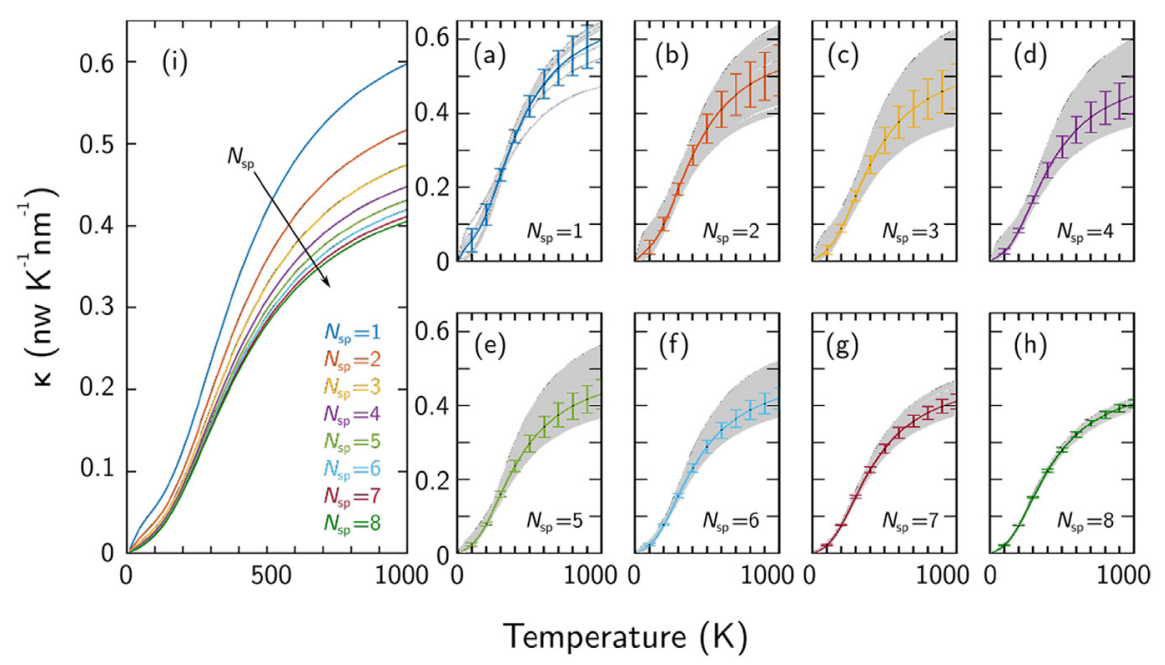

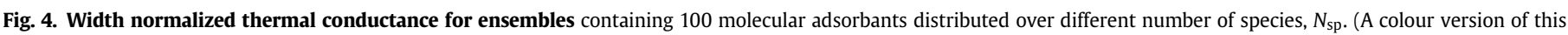
figure can be viewed online.) 
Table 1

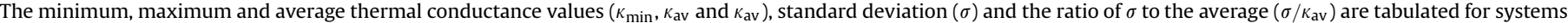

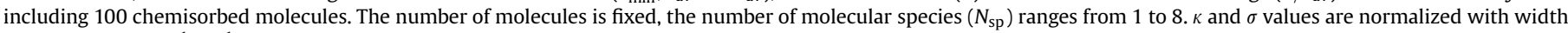
and given in $\mathrm{nW} \mathrm{K} \mathrm{K}^{-1} \mathrm{~nm}^{-1}$.

\begin{tabular}{|c|c|c|c|c|c|c|c|c|c|c|c|c|c|c|c|}
\hline \multirow[t]{2}{*}{$N_{\mathrm{sp}}$} & \multicolumn{5}{|l|}{$100 \mathrm{~K}$} & \multicolumn{5}{|l|}{$300 \mathrm{~K}$} & \multicolumn{5}{|l|}{$750 \mathrm{~K}$} \\
\hline & $\kappa_{\min }$ & $\kappa_{\max }$ & $\kappa_{\mathrm{av}}$ & $\sigma$ & $\sigma / \kappa_{\mathrm{av}}$ & $\kappa_{\min }$ & $\kappa_{\max }$ & $\kappa_{\mathrm{av}}$ & $\sigma$ & $\sigma / \kappa_{\mathrm{av}}$ & $\kappa_{\min }$ & $\kappa_{\max }$ & $\kappa_{\mathrm{av}}$ & $\sigma$ & $\sigma / \kappa_{\mathrm{av}}$ \\
\hline 1 & 0.020 & 0.109 & 0.056 & 0.030 & 0.527 & 0.213 & 0.257 & 0.233 & 0.015 & 0.066 & 0.356 & 0.450 & 0.419 & 0.015 & 0.064 \\
\hline 2 & 0.018 & 0.097 & 0.038 & 0.019 & 0.507 & 0.166 & 0.243 & 0.195 & 0.016 & 0.084 & 0.288 & 0.429 & 0.358 & 0.016 & 0.109 \\
\hline 3 & 0.017 & 0.083 & 0.030 & 0.012 & 0.406 & 0.146 & 0.219 & 0.177 & 0.013 & 0.075 & 0.260 & 0.423 & 0.328 & 0.013 & 0.108 \\
\hline 4 & 0.018 & 0.072 & 0.027 & 0.008 & 0.285 & 0.144 & 0.201 & 0.167 & 0.010 & 0.061 & 0.258 & 0.403 & 0.309 & 0.010 & 0.095 \\
\hline 5 & 0.018 & 0.051 & 0.025 & 0.005 & 0.190 & 0.144 & 0.191 & 0.160 & 0.008 & 0.048 & 0.257 & 0.380 & 0.297 & 0.008 & 0.078 \\
\hline 6 & 0.018 & 0.034 & 0.023 & 0.003 & 0.132 & 0.144 & 0.177 & 0.156 & 0.006 & 0.037 & 0.259 & 0.351 & 0.289 & 0.006 & 0.059 \\
\hline 7 & 0.019 & 0.028 & 0.022 & 0.002 & 0.089 & 0.144 & 0.165 & 0.153 & 0.004 & 0.025 & 0.261 & 0.317 & 0.284 & 0.004 & 0.041 \\
\hline 8 & 0.020 & 0.024 & 0.022 & 0.001 & 0.048 & 0.147 & 0.155 & 0.151 & 0.002 & 0.013 & 0.267 & 0.289 & 0.280 & 0.002 & 0.021 \\
\hline
\end{tabular}

\section{Conclusion}

A general scheme to engineer thermal transport properties of thin materials is proposed. Using the toy model, it is shown that antiresonances in the transmission spectra are determined by the molecular species and its coupling to the surface. Moreover, it is proven with the model that resistance summation rule is violated in phonon transport, which is due to the fact that relative positions of antiresonances play a major role in determining the total resistance. This fundamental feature of bosonic transport is shown to enable an engineering scheme for controlling thermal transport. At room temperature, $\kappa$ of a graphene sample with a fixed number of scatterers is reduced from $0.257 \mathrm{nW} \mathrm{K}^{-1} \mathrm{~nm}^{-1}$ to 0.144 $\mathrm{nW} \mathrm{K} \mathrm{K}^{-1} \mathrm{~nm}^{-1}$ by choosing a suitable combination of scatterers, where the resistance values of individual scatterers are almost the same. The proposed scheme can be used in a wide range of applications such as thermoelectrics and thermal management of microand nano-devices, where phonon transport plays a significant role.

\section{Acknowledgements}

Part of the computations are performed at TUBITAK ULAKBIM, High Performance and Grid Computing Center (TRUBA resources). HS acknowledges support from TÜBITAK $(113 C 032,115 F 445)$ and BAGEP program of Bilim Akademisi - the Science Academy, Turkey.

\section{Appendix A. Supplementary data}

Supplementary data related to this article can be found at https://doi.org/10.1016/j.carbon.2018.08.050.

\section{References}

[1] A. Balandin, S. Ghosh, W. Bao, I. Calizo, D. Teweldebrhan, F. Miao, C. Lau, Superior thermal conductivity of single-layer graphene, Nano Lett. 8 (3) (2008) 902-907, https://doi.org/10.1021/nl0731872.

[2] A. Balandin, Thermal properties of graphene and nanostructured carbon materials, Nat. Mater. 10 (8) (2011) 569-581, https://doi.org/10.1038/ nmat3064.

[3] E. Muñoz, J. Lu, B.I. Yakobson, Ballistic thermal conductance of graphene ribbons, Nano Lett. 10 (5) (2010) 1652-1656, https://doi.org/10.1021/ nl904206d.

[4] J. Haskins, A. Kınacı, C. Sevik, H. Sevinçli, G. Cuniberti, T. Çağın, Control of thermal and electronic transport in defect-engineered graphene nanoribbons, ACS Nano 5 (5) (2011) 3779-3787, https://doi.org/10.1021/nn200114p.

[5] W. Li, H. Sevinçli, G. Cuniberti, S. Roche, Phonon transport in large scale carbon-based disordered materials: implementation of an efficient order- $\mathrm{N}$ and real-space Kubo methodology, Phys. Rev. B 82 (4) (2010), https://doi.org/ 10.1103/PhysRevB.82.041410, 041410.

[6] W.J. Evans, L. Hu, P. Keblinski, Thermal conductivity of graphene ribbons from equilibrium molecular dynamics: effect of ribbon width, edge roughness, and hydrogen termination, Appl. Phys. Lett. 96 (20) (2010) 203112, https://doi.org/ 10.1063/1.3435465.

[7] N. Mingo, K. Esfarjani, D.A. Broido, D.A. Stewart, Cluster scattering effects on phonon conduction in graphene, Phys. Rev. B 81 (4) (2010), https://doi.org/
10.1103/PhysRevB.81.045408, 045408.

[8] H. Sevinçli, M. Brandbyge, Phonon scattering in graphene over substrate steps, Appl. Phys. Lett. 105 (15) (2014) 153108, https://doi.org/10.1063/1.4898066.

[9] H. Sevinçli, C. Sevik, T. Cağın, G. Cuniberti, A bottom-up route to enhance thermoelectric figures of merit in graphene nanoribbons, Sci. Rep. 3 (2013) 1228, https://doi.org/10.1038/srep01228.

[10] J.Y. Kim, J.C. Grossman, High-efficiency thermoelectrics with functionalized graphene, Nano Lett. (2015) 9050-9057, https://doi.org/10.1021/nl504257q.

[11] G.C. Solomon, D.Q. Andrews, R.P. Van Duyne, M.A. Ratner, When things are not as they seem: quantum interference turns molecular electron transfer "rules" upside down, J. Am. Chem. Soc. 130 (25) (2008) 7788-7789, https:// doi.org/10.1021/ja801379b.

[12] E.G. Emberly, G. Kirczenow, Antiresonances in molecular wires, J. Phys. Condens. Matter 11 (36) (1999) 6911.

[13] D. Nozaki, H. Sevinçli, S.M. Avdoshenko, R. Gutierrez, G. Cuniberti, A parabolic model to control quantum interference in T-shaped molecular junctions, Phys. Chem. Chem. Phys. 15 (33) (2013) 13951, https://doi.org/10.1039/c3cp44578j.

[14] T. Markussen, R. Stadler, K.S. Thygesen, The relation between structure and quantum interference in single molecule junctions, Nano Lett. 10 (10) (2010) 4260-4265, https://doi.org/10.1021/nl101688a.

[15] M. Ratner, A brief history of molecular electronics, Nat. Nanotechnol. 8 (6) (2013) 378-381.

[16] D. Nozaki, S.M. Avdoshenko, H. SevinÃÅli, R. Gutierrez, G. Cuniberti, Prediction of quantum interference in molecular junctions using a parabolic diagram: understanding the origin of Fano and anti- resonances, J. Phys. Conf. 427 (1) (2013), 012013.

[18] N. Zen, T.A. Puurtinen, T.J. Isotalo, S. Chaudhuri, I.J. Maasilta, Engineering thermal conductance using a two-dimensional phononic crystal, Nat. Commun. 5 (2014) 4435, https://doi.org/10.1038/ncomms4435.

[19] M. Maldovan, Phonon wave interference and thermal bandgap materials, Nat. Mater. 14 (7) (2015) 667-674, https://doi.org/10.1038/nmat4308.

[20] H. Han, L.G. Potyomina, A.A. Darinskii, S. Volz, Y.A. Kosevich, Phonon interference and thermal conductance reduction in atomic-scale metamaterials Phys. Rev. B 89 (18) (2014) 180301, https://doi.org/10.1103/ PhysRevB.89.180301.

[21] S. Xiong, K. Sääskilahti, Y.A. Kosevich, H. Han, D. Donadio, S. Volz, Blocking phonon transport by structural resonances in alloy-based nanophononic metamaterials leads to ultralow thermal conductivity, Phys. Rev. Lett. 117 (2) (2016), https://doi.org/10.1103/PhysRevLett.117.025503, 025503.

[22] Y. Yan, H. Zhao, Phonon interference and its effect on thermal conductance in ring-type structures, J. Appl. Phys. 111 (11) (2012) 113531, https://doi.org/ 10.1063/1.4729297.

[23] D. Ma, H. Ding, H. Meng, L. Feng, Y. Wu, J. Shiomi, N. Yang, Nano-crossjunction effect on phonon transport in silicon nanowire cages, Phys. Rev. B 94 (16) (2016) 165434, https://doi.org/10.1103/PhysRevB.94.165434.

[24] N. Mingo, Green's function methods for phonon transport through nanocontacts, in: S. Volz (Ed.), Thermal Nanosystems and Nanomaterials, No. 118 in Topics in Applied Physics, Springer Berlin Heidelberg, 2009, pp. 63-94.

[25] H. Sevinçli, S. Mukhopadhyay, R.T. Senger, S. Ciraci, Dynamics of phononic dissipation at the atomic scale: dependence on internal degrees of freedom, Phys. Rev. B 76 (20) (2007) 205430.

[26] D. Porezag, T. Frauenheim, T. Köhler, G. Seifert, R. Kaschner, Construction of tight-binding-like potentials on the basis of density-functional theory: application to carbon, Phys. Rev. B 51 (1995) 12947-12957.

[27] G. Seifert, D. Porezag, T. Frauenheim, Calculations of molecules, clusters, and solids with a simplified lcao-dft-lda scheme, Int. J. Quant. Chem. 58 (2) (1996) $185-192$.

[28] M. Elstner, D. Porezag, G. Jungnickel, J. Elsner, M. Haugk, T. Frauenheim, S. Suhai, G. Seifert, Self-consistent-charge density-functional tight-binding method for simulations of complex materials properties, Phys. Rev. B 58 (1998) 7260-7268.

[29] B. Aradi, B. Hourahine, T. Frauenheim, Dftb+, a sparse matrix-based implementation of the dftb method, J. Phys. Chem. 111 (26) (2007) 5678-5684

[30] A. Togo, I. Tanaka, First principles phonon calculations in materials science, Scripta Mater. 108 (2015) 1-5. 
[31] L.G.C. Rego, G. Kirczenow, Quantized thermal conductance of dielectric quantum wires, Phys. Rev. Lett. 81 (1) (1998) 232-235, https://doi.org/ 10.1103/PhysRevLett.81.232.

[32] S. Datta, Electronic Transport in Mesoscopic Systems, Cambridge Studies in Semiconductor Physi, Cambridge University Press, 1997.

[33] T. Markussen, R. Rurali, A.-P. Jauho, M. Brandbyge, Scaling theory put into practice: first-principles modeling of transport in doped silicon nanowires, Phys. Rev. Lett. 99 (2007), 076803.
[34] I. Savić, N. Mingo, D.A. Stewart, Phonon transport in isotope-disordered carbon and boron-nitride nanotubes: is localization observable? Phys. Rev. Lett. 101 (2008) 165502.

[35] J. Wang, L. Li, J.-S. Wang, Tuning thermal transport in nanotubes with topological defects, Appl. Phys. Lett. 99 (9) (2011), 091905.

[36] D.A. Stewart, I. Savić, N. Mingo, First-principles calculation of the isotope effect on boron nitride nanotube thermal conductivity, Nano Lett. 9 (1) (2009) $81-84$. 